\title{
Restoring vision using optogenetics without being blind to the risks
}

\author{
Alexander R. Harris ${ }^{1}$. Frederic Gilbert ${ }^{2}$ \\ Received: 10 August 2021 / Revised: 12 October 2021 / Accepted: 25 October 2021 / Published online: 1 November 2021 \\ (c) The Author(s), under exclusive licence to Springer-Verlag GmbH Germany, part of Springer Nature 2021
}

\begin{abstract}
Retinit is pigmentosa is an incurable degenerative disease that causes loss of light-sensitive cells in the retina and leads to severe vision impairment. The development of optogenetics has created great hype around its potential to treat retinitis pigmentosa by the introduction of light-sensitive proteins into other neural cells in the retina. The first-in-human studies of optogenetic treatment for this disease have recently been reported (NCT02556736 and NCT03326336). The treatment involves irreversible gene therapy and requires access to specially designed goggles to deliver light to the treated eye. These highly innovative and high-profile clinical trials raise numerous ethical issues that must be addressed during the early phases of research and clinical testing to ensure trial participants are treated fairly and can provide appropriate informed consent.
\end{abstract}

Keywords Ethics $\cdot$ Clinical trial $\cdot$ Informed consent $\cdot$ Optogenetics $\cdot$ Retinitis pigmentosa

\section{Key messages}

- Optogenetics has the potential to deliver partial recovery of visual function to people with severe vision impairment. It is a form of gene therapy that involves irreversible changes to patients.

- The current approach to clinical trials is that participants are placed at risk testing the safety and efficacy of novel treatments; they should not expect any immediate therapeutic benefits, but may in the future if the trial is successful. In the case of irreversible treatments, participants lose their rights to withdraw from a trial and potentially any future therapeutic benefit.

- Optogenetics clinical trial participants must be made aware of the large range of short and long term risks to ensure they can provide appropriate informed consent.

- Clinical trial sponsors must ensure they can provide ongoing support and resource access to participants undergoing irreversible treatments.

Alexander R. Harris

alexrharris@gmail.com

$\bowtie$ Frederic Gilbert frederic.gilbert@utas.edu.au

1 Aikenhead Centre for Medical Discovery, ARC Centre of Excellence for Electromaterials Science, Faculty of Medicine, Dentistry and Health Sciences, University of Melbourne, Melbourne, VIC 3010, Australia

2 School of Humanities, University of Tasmania, Hobart, Australia

\section{Introduction}

Optogenetics consists of introducing light-sensitive proteins (opsins) into cells, such as neurons, allowing their behaviour to be controlled optically. As such, it presents unprecedented potential to control tissue function through the application of light to genetically modified target cells [1]. The first human trial testing optogenetics dates from 2016 (clinicaltrials. gov NCT02556736). This novel invasive technology shows great potential for medical application through treatment 
of neurological and psychiatric conditions such as retinitis pigmentosa (RP), Parkinson's disease (PD), addiction, schizophrenia, autism, and depression. There are ongoing ethical debates about which population is best targeted for optogenetics [2, 3], usage for memory modification [4-7], and whether some ethical concerns linked to testing optogenetics in humans belong to the realm of speculative ethics $[4,8,9]$.

A recent ground-breaking study has established further efficacy and safety information of optogenetics in humans. This new demonstration of partial recovery of visual function in a blind patient after optogenetic therapy is an important step in treating RP and potentially other disorders [10] and indicates optogenethics is crucial to the advancement of the technology. However, the original research and subsequent clinical trials of this technology raised numerous safety and ethical issues, many persistent ones, while other new issues have arisen [3]. While there are general guidelines on what risks gene therapy-trial participants should be informed about [11], the decision is almost always determined by the researchers, with feedback from an Institutional Review Board [12]. As a result, the informed consent covering health risks may only focus on potential changes to tissue, cancer formation, transfer of genes to future progeny, or infection of close contacts. These are generally well-studied risks that are highly unlikely to occur, allowing researchers to portray the trial as very low-risk. It may downplay other significant risks to trial participants due to the unknown impact of the treatment. Futhermore, as observed with other experimental invasive technologies [13], positive reporting of a clinical trial in mainstream media often ignores the risks and unproven nature of the treatment which may further bias participants' view of their inclusion. It is critical that risks associated with participation in an optogenetics trial (i.e. NCT03326336) are appropriately addressed during the early clinical trial phases to ensure patients are treated fairly, and have sufficient informed consent, based on the short- and long-term risks and benefits of the potential therapy.

\section{Treatment risks}

The current trial uses an adeno-associated viral vector (AAV) to deliver the genes coding for the optogenetic protein (NCT03326336). Cells expressing opsins are not sufficiently light-sensitive on their own to provide visual function; the trial participant must also wear goggles that deliver a high-intensity image to the treated retina. The primary outcome of the phase $1 / 2 \mathrm{a}$ trial is to determine the safety and tolerability of a single viral dose over 1 year. Secondary measures include visual acuity assessments and evaluation of immune response up to 1 year. The initial cohort is estimated at 15 participants with varying viral vector dosage. While this provides crucial safety and efficacy information, protein expression and function can vary between recipients receiving equivalent dosages. Participants who receive some therapeutic response may only have activation in small regions of the retina, limiting their field of view. It is also unknown how long protein expression will persist or if phototoxic effects will occur, so a therapeutic dose may degrade over time. As a result, some patients may require multiple doses to achieve a therapeutic response. This raises the risk of a potential immune response which is not being assessed in the current trial. In an attempt to minimize this risk, the current trial precludes participants who have previously received gene therapy (typical for all gene therapy trials). It also only treated the worse-seeing eye, to minimize the impact of potential adverse events. However, if these participants receive any therapeutic benefit from the trial, they may be precluded from being inoculated over a wider region of the treated eye or in the other eye.

AAVs are also being used in other treatments, including the voretigene neparvovec-rzyl gene therapy for RPE65 mutation-associated inherited retinal disease [14] and the Oxford-AstraZeneca and Johnson \& Johnson COVID-19 vaccines. While these particular treatments use different AAV serotypes, the increased usage of AAVs in biomedical treatments raises the risk of participants having repeated exposure to these vectors, increasing the chance of a harmful immune response. This could prevent people participating in a trial or receiving treatment, limiting their future benefit. It may also have public health impacts if large numbers of people become immune to certain viral vectors and are unable to receive vaccines. It would therefore be unethical to run a new clinical trial with a different viral vector or opsin for the sole purpose of novelty if one has already been shown to be safe and effective. It may therefore be unethical to use AAV serotype $2.7 \mathrm{~m} 8$ for the optogenetics trial when the voretigene neparvovec-rzyl retinal gene therapy has already undergone dosage studies and received FDA approval using AAV serotype 2. One of the side effects of the AAV COVID19 vaccines has been thrombosis with thrombocytopenia [15]. Another serotype, Ad5, may also increase the risk of contracting HIV [16]. While these particular side effects are unlikely with the optogenetics treatment, it highlights the variability and unpredictability of side effects that can occur from gene therapy. There is potential for these types of low-risk events occurring from the retinal optogenetics therapy, which are unlikely to be detected in such research trials given the small number of participants. These types of issues may not be detected until well passed the 1 year trial completion. Inclusion and exclusion of some patients with comorbidities, and/or medical predispositions, should guide recruitment eligibility. As trial stages progress and 
new knowledge is obtained, the need to remove some vulnerable participants may be critical.

\section{Right to withdraw}

A fundamental safety guarantee of research trials is the capacity of participants to withdraw at any time before and during the trial. The current trial (NCT03326336) uses viral dose-escalation to evaluate its safety and tolerability-which satisfies common drug dosage protocols enabling withdrawal or termination of a trial; patients enrolling in a trial testing optogenetics might be prevented in exercising their right to withdraw after inoculation. Optogenetics is limited in terms of reversibility of the procedure, and attempting to reverse the implantation may lead to severe harm (e.g. surgical trauma and scar tissue formation). What makes it more difficult to withdraw from the trial is that in most cases, it is likely that patients will be facing a 'last chance' scenario: putting them in an irreversible situation. Even if the treated patient is in a stable condition at the conclusion of the trial, the treated cells cannot be substituted for a superior treatment in the future. For instance, participants in this trial may be prevented from being treated with other optogenetic methods which may be more effective (e.g. Allergan NCT02556736) or other types of therapies such as a bionic eye (e.g. Second Sight Medical Products) or stem cell therapy. Therefore, participating in an optogenetics trial may cause patients to lose their opportunity and eligibility for future treatment. Ethically speaking, the inability to obtain treatment due to potential harm and irreversibility of a treatment is only one concern [17]. The key point here is not that patients missed out on another treatment, but because they participated in an experimental optogenetics trial, they missed out on better treatment in the future.

\section{Sponsor obligations}

Given the permanent nature of invasive optogenetic interventions, post-trial obligations raise enormous safety and moral questions. What happens if the trial needs to be terminated due to unexpected reasons (i.e. financial, regulations, etc.)? The first company which tested optogenetics in humans, Retrosense, was bought by Allergan in 2016. When new investors are introduced into a protocol, it is never clear whether a trial will remain active, and if not, what will happen to the irreversibly inoculated participants. Will participants still have access to the goggles required for visual function? Even if a protocol is financially robust and remains active, there are unknown consequences. What happen if a participant has received therapeutic benefits from the trial but the protein expression degrades or inoculated cells do not survive, returning them to blindness and preventing any future treatment? There is potential for participants to experience unanticipated psychological harms at the end of the trial by virtue of treatment degradation or withdrawal by loss of access to the goggles [18-20]. Where possible, participants receiving a therapeutic benefit from the optogenetics trial should be offered ongoing access to the goggles and other facilities required for maintaining vision. Any protocol involving irrevocable and permanent change to the nervous system, not just the optogenetics trial, should account for the safety and ethics of an early terminating trial, especially when irreversible harms may appear in small numbers of cases long after termination. Post-trial access to medical support will likely be challenging given the limited number of clinics capable of dealing with innovative optogenetic trials. Participants may be forced to attend unique, specialized laboratories, affecting future lifestyle and residency options. They may also be dependent on highly trained individuals that are no longer employed on the trial or do not have access to the necessary facilities. Clinical teams and patients should be prepared to address a potential ending of the trial. Equally important, many ethical issues may only become apparent one or two decades after treatment, as with other technologies which were also in first-in-human trial at some point. For instance, deep brain stimulation, which was an experimental invasive brain surgery in the 1990s targeting PD, now appears to be linked to an increase of life expectancy of PD's patients, according to recent longitudinal follow-up studies [21-23]. However, this longitudinal medical benefit does not come without attendant negative consequences, such as inducing iatrogenic harms that were not initially expected when the trials were first conducted [24, 25]. Should optogenetics become an effective and safe treatment for neurodegenerative diseases, there is a strong chance it will impact life expectancy and disease trajectory, and therefore has a potential for inducing iatrogenic harms to patients.

Beyond post-trial responsibilities and accountability for participating companies and clinicians, questions of access and justice from potential optogenetic treatments must be anticipated. How should optogenetic treatments be allocated given a scarcity of resources? Will patients only located in advantageous socio-economic groups be the beneficiary of the treatment? Where possible, distribution of optogenetic interventions should be made by the public health care system to ensure a fair and equitable approach over all strata of the population, eliminating potential injustice in treatment administration. 


\section{Conclusion}

As a reminder, the false hope of treatment may be synonymous with participant recruitment in these types of experimental optogenetics trials. Due to the progressive nature of $\mathrm{RP}$, many patients may have exhausted all standard therapeutic options, and consider an experimental optogenetics trial as a last resort treatment. Patients with RP are at increased risk of conflating participation in a clinical trial with accessing novel medical treatment, a well-known phenomenon during experimental stage trials identified as therapeutic misconceptions [26]. Desperate patients may not realize the disadvantages of participating in an experimental optogenetics trial, because the design of the protocol focuses on safety rather than effective therapeutic outcomes (i.e. aim at non-therapeutic endpoints, not at the needs or interests of participants). While there are many novel technologies entering clinical trial which may have profound benefits to individuals and society, clinicians and trial participants must not be blind to their potential short- and long-term risks of harm.

\section{Declarations}

Ethics approval This article does not contain any studies with human participants or animals performed by any of the authors.

Conflict of interest The authors declare no competing interests.

\section{References}

1. Buchen L (2010) Neuroscience: illuminating the brain. Nature 465:26-28. https://doi.org/10.1038/465026a

2. Maslen H, Savulescu J (2014) First phase 1 optogenetic trials should be conducted in people who are dying. AJOB Neurosci 5:16-18. https://doi.org/10.1080/21507740.2014.911216

3. Gilbert F, Harris AR, Kapsa RMI (2014) Controlling brain cells with light: ethical considerations for optogenetic clinical trials. AJOB Neurosci 5:3-11. https://doi.org/10.1080/21507740. 2014.911213

4. Adamczyk AK, Zawadzki P (2020) The memory-modifying potential of optogenetics and the need for neuroethics. NanoEthics 14:207-225. https://doi.org/10.1007/s11569-020-00377-1

5. Zawadzki P, Adamczyk AK (2021) Personality and authenticity in light of the memory-modifying potential of optogenetics. Am J Bioeth - Neurosci. https://doi.org/10.1080/21507740.2020. 1866097

6. Tan SZK, Lim LW (2020) A practical approach to the ethical use of memory modulating technologies. BMC Med Ethics 21:89. https://doi.org/10.1186/s12910-020-00532-z

7. Gilbert F, Harris AR, Kidd M (2021) Burnt in your memory or burnt memory? Ethical issues with optogenetics for memory modification. AJOB Neurosci 12:22-24. https://doi.org/10. 1080/21507740.2020.1866104
8. Gilbert F, Goddard E (2014) Thinking ahead too much: speculative ethics and implantable brain devices. AJOB Neurosci 5:49-51. https://doi.org/10.1080/21507740.2013.863252

9. Müller S, Walter H (2014) Neither speculative nor narrowminded ethics is needed for optogenetics-based DBS in psychiatry and neurology. AJOB Neurosci 5:12-14. https://doi.org/ $10.1080 / 21507740.2014 .911780$

10. Sahel J-A, Boulanger-Scemama E, Pagot C et al (2021) Partial recovery of visual function in a blind patient after optogenetic therapy. Nat Med. https://doi.org/10.1038/s41591-021-01351-4

11. Nih (2014) Informed consent guidance for human gene transfer trials subject to the NIH guidelines for research involving recombinant or synthetic nucleic acid molecules. https://osp.od. nih.gov/wp-content/uploads/2014/10/IC2013.pdf. Accessed 12 Oct 2021

12. Desine S, Hollister BM, Abdallah KE et al (2020) The meaning of informed consent: genome editing clinical trials for sickle cell disease. AJOB Empir Bioeth 11:195-207. https://doi.org/ 10.1080/23294515.2020.1818876

13. Gilbert F, Pham C, Viana JNM, Gillam W (2019) Increasing brain-computer interfaces media depictions: pressing ethical concerns. Brain-Computer Interfaces. 6(3):49-70. https://doi. org/10.1080/2326263X.2019.1655837

14. Ciulla TA, Hussain RM, Berrocal AM, Nagiel A (2020) Voretigene neparvovec-rzyl for treatment of RPE65-mediated inherited retinal diseases: a model for ocular gene therapy development. Expert Opin Biol Ther 20:565-578. https://doi.org/10. 1080/14712598.2020.1740676

15. Greinacher A, Thiele T, Warkentin TE et al (2021) Thrombotic thrombocytopenia after ChAdOx1 nCov-19 vaccination. N Engl J Med. https://doi.org/10.1056/NEJMoa2104840

16. Perreau M, Pantaleo G, Kremer EJ (2008) Activation of a dendritic cell-T cell axis by Ad5 immune complexes creates an improved environment for replication of HIV in T cells. J Exp Med 205:2717-2725. https://doi.org/10.1084/jem.20081786

17. Gilbert F, Harris AR, Kapsa RMI (2012) Efficacy testing as a primary purpose of phase 1 clinical trials: is it applicable to first-in-human bionics and optogenetics trials? AJOB Neurosci 3:20-22. https://doi.org/10.1080/21507740.2012.666323

18. Lawton J, Blackburn M, Rankin D et al (2019) Broadening the debate about post-trial access to medical interventions: a qualitative study of participant experiences at the end of a trial investigating a medical device to support type 1 diabetes selfmanagement. AJOB Empir Bioeth 10:100-112. https://doi.org/ 10.1080/23294515.2019.1592264

19. Lázaro-Muñoz G, Yoshor D, Beauchamp MS et al (2018) Continued access to investigational brain implants. Nat Rev Neurosci 19:317-318. https://doi.org/10.1038/s41583-018-0004-5

20. Gilbert F (2015) Self-estrangement \& deep brain stimulation: ethical issues related to forced explantation. Neuroethics 8:107114. https://doi.org/10.1007/s12152-014-9224-1

21. Lau B, Meier N, Serra G, et al (2019) Axial symptoms predict mortality in patients with Parkinson disease and subthalamic stimulation. Neurology 92:e2559 LP-e2570. https://doi.org/10. 1212/WNL.0000000000007562

22. Ngoga D, Mitchell R, Kausar J et al (2014) Deep brain stimulation improves survival in severe Parkinson's disease. J Neurol Neurosurg Psychiatry 85:17-22. https://doi.org/10.1136/ jnnp-2012-304715

23. Bang Henriksen M, Johnsen EL, Sunde N et al (2016) Surviving 10 years with deep brain stimulation for Parkinson's disease - a follow-up of 79 patients. Eur J Neurol 23:53-61. https://doi.org/ 10.1111/ene. 12614

24. Gilbert F, Lancelot M (2021) Incoming ethical issues for deep brain stimulation: when long-term treatment leads to a "new 
form of the disease.' J Med Ethics 47:20 LP - 25. https://doi. org/10.1136/medethics-2019-106052

25. Sankary LR, Ford PJ, Machado AG et al (2019) Deep brain stimulation at end of life: clinical and ethical considerations. J Palliat Med 23:582-585. https://doi.org/10.1089/jpm.2019.0129

26. Appelbaum PS, Roth LH, Lidz CW et al (1987) False hopes and best data: consent to research and the therapeutic misconception. Hastings Cent Rep 17:20-24. https://doi.org/10.2307/3562038
Publisher's note Springer Nature remains neutral with regard to jurisdictional claims in published maps and institutional affiliations. 